Quasi-exact solvability, resonances and trivial monodromy in ordinary differential equations

This article has been downloaded from IOPscience. Please scroll down to see the full text article.

2012 J. Phys. A: Math. Theor. 45444013

(http://iopscience.iop.org/1751-8121/45/44/444013)

View the table of contents for this issue, or go to the journal homepage for more

Download details:

IP Address: 192.135.19.98

The article was downloaded on 26/10/2012 at 10:53

Please note that terms and conditions apply. 


\title{
Quasi-exact solvability, resonances and trivial monodromy in ordinary differential equations
}

\author{
Patrick Dorey $^{1}$, Clare Dunning ${ }^{2}$ and Roberto Tateo ${ }^{3}$ \\ ${ }^{1}$ Department of Mathematical Sciences, University of Durham, Durham DH1 3LE, UK \\ ${ }^{2}$ SMSAS, University of Kent, Canterbury CT2 7NF, UK \\ ${ }^{3}$ Dipartimento di Fisica and INFN, Università di Torino, Via P Giuria 1, I-10125 Torino, Italy \\ E-mail: p.e.dorey@durham.ac.uk,t.c.dunning@kent.ac.uk and tateo@to.infn.it
}

Received 30 March 2012, in final form 20 August 2012

Published 23 October 2012

Online at stacks.iop.org/JPhysA/45/444013

\begin{abstract}
A correspondence between the sextic anharmonic oscillator and a pair of thirdorder ordinary differential equations is used to investigate the phenomenon of quasi-exact solvability for eigenvalue problems involving differential operators with order greater than 2. In particular, links with Bender-Dunne polynomials and resonances between independent solutions are observed for certain secondorder cases, and extended to the higher-order problems.
\end{abstract}

This article is part of a special issue of Journal of Physics A: Mathematical and Theoretical devoted to 'Quantum physics with non-Hermitian operators'.

PACS numbers: 03.65.-Ge, 11.15.Tk, 11.25.HF, 11.55.DS

\section{Introduction}

Despite the simplicity of one-dimensional quantum-mechanical systems, full solvability is very much an exception rather than the rule. The archetypal example is the harmonic oscillator, for which exact solvability breaks down completely under almost any kind of perturbation. Nevertheless, Turbiner [1] found the surprising fact that in some cases, corresponding to certain multi-parameter families of second-order differential eigenvalue problems, there are regions of the parameter space for which a finite subset of the spectrum can be found algebraically. Turbiner and Ushveridze [2] dubbed these models quasi-exactly solvable, or QES. For the QES problems of [1], and almost all other examples, the differential operators $\mathcal{H}$ act invariantly in a finite dimensional subspace $\mathcal{P}_{n+1}$ spanned, possibly after a gauge transformation, by the monomials $1, x, \ldots, x^{n}$.

It is natural to ask whether similar ideas might apply to eigenvalue problems involving higher-order differential operators. Turbiner has given a general classification of differential operators of order $k$ with finite dimensional subspace $\mathcal{P}_{n+1}$ [3] for which $n+1$ eigenfunctions have the form of a polynomial of order at most $n$. Examples of higher-order QES operators arising in multi-mode bosonic Hamiltonians relevant to nonlinear optics may be found in [4-8]. 
In this paper we consider third-order problems, motivated by a link between a specific family of third-order differential equations and the Schrödinger equation for the sextic anharmonic oscillator that was discovered and explored in $[9,12]$. By tuning the parameters so that the second-order eigenproblem is at the points at which its spectrum is QES, the corresponding isospectral third-order eigenproblem must also be QES. It turns out that the quasi-exact solvability of these third-order differential equations is rather subtle, and even though a finite subset of eigenvalues can be found exactly, the most natural ansatz for the corresponding eigenfunctions turns out not to hold. Nevertheless, we shall exhibit a number of interesting properties of these and related second-order eigenproblems, and show in particular that the QES eigenfunctions are the generating functions for sets of Bender-Dunne polynomials whose zeros correspond to the QES eigenvalues.

\section{Isospectral second and third order eigenproblems}

Turbiner showed [1] that the sextic potential

$$
\mathcal{H}_{2}(\alpha, l) \psi(x) \equiv\left[-\frac{\mathrm{d}^{2}}{\mathrm{~d} x^{2}}+x^{6}+\alpha x^{2}+\frac{l(l+1)}{x^{2}}\right] \psi(x)=E \psi(x),
$$

with regular boundary conditions imposed on the positive real axis at $x=0$ and $x \rightarrow \infty$ by requiring for $l>-1 / 2$

$$
\left.\psi\right|_{x \rightarrow 0}=x^{l+1}\left(1+O\left(x^{2}\right)\right), \quad \psi(x)=O\left(x^{-3 / 2-\alpha / 2} \mathrm{e}^{-x^{4} / 4}\right) \text { as } x \rightarrow \infty,
$$

is quasi-exactly solvable along the lines

$$
\alpha=\alpha_{J}=-(2 l+1+4 J), \quad J=1,2,3, \ldots
$$

The operator $\mathcal{H}_{2}\left(\alpha_{J}, l\right)$ is quasi-exactly solvable because it acts invariantly in the finite dimensional subspace $\left\langle f_{0}, f_{1}, \ldots, f_{J-1}\right\rangle$ where $f_{n}(x)=x^{l+1} \exp \left(-x^{4} / 4\right) x^{2 n}$. The gauge transformation $x^{-l-1} \exp \left(x^{4} / 4\right) \mathcal{H}_{2} x^{l+1} \exp \left(-x^{4} / 4\right)$ and variable change $x^{2}=w$ transforms the Schrödinger equation (2.1) into a second-order differential equation which acts invariantly on $\mathcal{P}_{J} \equiv\left\langle\mid, w, \ldots, w^{J-1}\right\rangle[1]$.

A convenient way to handle the quasi-exact solvability of (2.1) is through the BenderDunne polynomials introduced in [10]. The idea is to write a solution to (2.1) in the following factorized form

$$
\psi(x)=\mathrm{e}^{-x^{4} / 4} x^{l+1} \sum_{n=0}^{\infty}\left(-\frac{1}{4}\right)^{n} \frac{P_{n}(E, \alpha, l)}{n ! \Gamma(n+l+3 / 2)} x^{2 n} .
$$

For (2.4) to solve (2.1), the coefficients $P_{n}$ must satisfy the recursion relation

$$
P_{n}(E)=E P_{n-1}(E)+16(n-1)(n-j-1)(n+l-1 / 2) P_{n-2}(E) \quad(n \geqslant 1)
$$

with $j=j(\alpha, l)=-(\alpha+2 l+1) / 4$ and $P_{0}(E)=1$. From (2.5), $P_{1}=E$, and $P_{n}$ is a polynomial of degree $n$ in $E$, known as a Bender-Dunne polynomial. As long as $l \neq-n-3 / 2$ for any $n \in \mathbb{Z}^{+}$, (2.4) will yield an everywhere-convergent series solution to (2.1). This solution automatically satisfies the boundary condition at the origin, but at general values of $E$, it will grow exponentially as $x \rightarrow \infty$. However, if $\alpha$ and $l$ are such that $j(\alpha, l)=J$ is a positive integer, the second term on the rhs of (2.5) vanishes at $n=J+1$, and all subsequent $P_{n}$ factorize:

$P_{n+J}\left(E, \alpha_{J}, l\right)=P_{J}\left(E, \alpha_{J}, l\right) Q_{n}\left(E, \alpha_{J}, l\right) \quad\left(n>0, J=-\left(\alpha_{J}+2 l+1\right) / 4 \in \mathbb{N}\right)$. 
Thus, if $P_{J}(E)$ vanishes then so do all $P_{n \geqslant J}(E)$ and the series (2.4) terminates, automatically giving a normalizable solution to (2.1). The $J$ roots of $P_{J}(E)$ are the $J$ exactly-solvable energy levels for the model. For $J=1$ and $J=2$, the exactly-solvable eigenvalues are

$$
J=1: E_{0}=0, J=2: E_{ \pm}= \pm 2 \sqrt{2} \sqrt{3+2 l} .
$$

For all real values of the parameter $\alpha$ and $l>-1 / 2$ the sextic eigenproblems (2.1), (2.2) have up to scaling exactly the same eigenvalues as a family of third-order eigenproblems $[9,12]$. The relevant third-order differential equation is

$$
\mathcal{H}_{3} \phi(x) \equiv\left[\frac{\mathrm{d}^{3}}{\mathrm{~d} x^{3}}+x^{3}+\frac{L}{x^{3}}-G\left(\frac{1}{x^{2}} \frac{\mathrm{d}}{\mathrm{d} x}-\frac{1}{x^{3}}\right)\right] \phi(x)=\bar{E} \phi(x)
$$

where $^{4}$

$$
\begin{aligned}
& G=2-\left(g_{0} g_{1}+g_{0} g_{2}+g_{1} g_{2}\right), \\
& L=-2-g_{0} g_{1} g_{2}+\left(g_{0} g_{1}+g_{0} g_{2}+g_{1} g_{2}\right), \\
& g_{0}+g_{1}+g_{2}=3,
\end{aligned}
$$

and the boundary conditions on the positive real axis are

$$
\left.\phi\right|_{x \rightarrow 0}=x^{g_{1}}\left(1+O\left(x^{3}\right)\right), \quad \phi(x)=O\left(x^{-1} \mathrm{e}^{-x^{2} / 2}\right) \text { as } x \rightarrow \infty
$$

with $g_{0}<g_{1}<g_{2}$. The asymptotic condition ensures the other two possible behaviours of the solution at infinity are ruled out. The isospectrality of $\mathcal{H}_{2}, \mathcal{H}_{3}$ with boundary conditions (2.2), (2.12) respectively was first discussed in [9] for $l=0$ and, with the help of results from [11], generalized to $l \neq 0$ in [12]. The result is that the eigenvalues $E, \bar{E}$ associated to $\mathcal{H}_{2}, \mathcal{H}_{3}$ satisfy

$$
\bar{E}=E / \kappa, \quad \kappa=4 /(3 \sqrt{3}),
$$

whenever the parameters $\{\alpha, l\}$ and $\left\{g_{0}, g_{1}, g_{2}\right\}$ in the two models are related as

$$
\alpha=2\left(2-g_{0}-g_{2}\right), \quad l=\left(2 g_{2}-3-2 g_{0}\right) / 6,
$$

and

$$
g_{0}=(1-\alpha-6 l) / 4, \quad g_{1}=(1+\alpha / 2), \quad g_{2}=(7-\alpha+6 l) / 4 .
$$

The result was obtained by showing that the associated spectral determinants-functions constructed to vanish at the eigenvalues - are proportional. By analytical continuation from $l$ to $-1-l$, the isospectrality result also extends to $l \leqslant-1 / 2[9,12]$.

If $\alpha$ is now tuned to the quasi-exactly solvable points $\alpha=-(4 J+2 l+1)$ of $\mathcal{H}_{2}$ for positive integer $J$, then via the isospectrality (2.13) the third-order problem $\mathcal{H}_{3}$ with

$$
g_{0}=1 / 2+J-l, \quad g_{1}=1 / 2-2 J-l, \quad g_{2}=2+J+2 l
$$

has a hidden QES sector and $J$ eigenvalues can be found exactly. Since the isospectrality proof relates the eigenvalues and makes no conclusions about the eigenfunctions, we cannot immediately state that $\mathcal{H}_{3}$ at the points (2.16) is itself 'fully' quasi-exactly solvable, if for full quasi-exact solvability one would insist on being able to find algebraically not only a subset of the eigenvalues but also the corresponding eigenfunctions. One might expect that $\mathcal{H}_{3}$ acts invariantly in a finite-dimensional subspace spanned by functions of the form $x^{g_{1}} \exp \left(-x^{2} / 2\right) x^{n}$. If that is the case, the QES eigenfunctions will take a simple factorized form generalizing (2.4). We shall show that this is not in general the case.

4 In equations (2.9) and (2.10) we have corrected an overall sign typo in the corresponding equations of [12]. 
In [13] and [14], the spectral link between $\mathcal{H}_{2}$ and $\mathcal{H}_{3}$ was naturally extended to the adjoint operator $\mathcal{H}_{3}^{\dagger}$ to $(2.8)$ :

$\mathcal{H}_{3}^{\dagger} \chi^{\dagger}(x) \equiv\left[\frac{\mathrm{d}^{3}}{\mathrm{~d} x^{3}}-x^{3}+\frac{L^{\dagger}}{x^{3}}-G^{\dagger}\left(\frac{1}{x^{2}} \frac{\mathrm{d}}{\mathrm{d} x}-\frac{1}{x^{3}}\right)\right] \chi^{\dagger}(x)=-\bar{E} \chi^{\dagger}(x)$

where $G^{\dagger} \equiv G, L^{\dagger} \equiv-L$. The appropriate boundary conditions are specified by

$$
\left.\chi^{\dagger}\right|_{x \rightarrow 0}=x^{g_{0}^{\dagger}}\left(1+O\left(x^{3}\right)\right),\left.\chi^{\dagger}\right|_{x \rightarrow \infty} \rightarrow 0\left(g_{0}^{\dagger}=2-g_{0}\right) .
$$

It is important to note that the eigenfunctions $\phi_{n}$ of $\mathcal{H}_{3}$ and the eigenfunctions $\chi_{n}^{\dagger}$ of $\mathcal{H}_{3}^{\dagger}$ with eigenvalues $\bar{E}_{n}$ and $-\bar{E}_{n}$ respectively are substantially different functions, not related to each other by simple conjugation. Provided the roots of the indicial equation are ordered as $g_{0}<g_{1}<g_{2}$ then for $g_{1}>-1 / 2$ the $\phi_{n}$ s are square integrable on $\mathbb{R}^{+}$,

$$
\lim _{x \rightarrow 0} \chi_{n}^{\dagger}(x) \phi_{m}(x)=O\left(x^{2-g_{0}+g_{1}}\right) \rightarrow 0, \quad \lim _{x \rightarrow \infty} \chi_{n}^{\dagger}(x) \phi_{m}(x) \rightarrow 0,
$$

and the sets $\left\{\chi_{n}^{\dagger}\right\}$ and $\left\{\phi_{n}\right\}$ can always be normalized such that

$$
\langle n \mid m\rangle=\int_{0}^{\infty} \chi_{n}^{\dagger}(x) \phi_{m}(x) \mathrm{d} x=\delta_{n, m} .
$$

The properties described above are reminiscent of the well-studied properties of non-selfadjoint spectral problems and biorthogonal systems in quantum mechanics. These systems were introduced and studied in the early days of quantum mechanics, and were more recently revisited in the context of $\mathcal{P} \mathcal{T}$-symmetric quantum mechanical models [15]. The reader is addressed to [16] for a recent review of this material.

\section{Bender-Dunne polynomials and projective triviality}

It is natural to ask whether a simple factorization similar to (2.4) also characterizes the exactly-solvable energy levels of $\mathcal{H}_{3}$ and $\mathcal{H}_{3}^{\dagger}$. To answer this question we first identify a simple necessary condition for the existence of a factorizable solution to equation (2.1) of the form

$$
\psi(x)=x^{l+1} \mathcal{P}_{2 J-2}(x, E) \mathrm{e}^{-x^{4} / 4},
$$

where $\mathcal{P}_{2 J}(x, E)$ is a polynomial of order $2 J$ in $x$. The large- $x$ behaviour of $\psi$ should match the general WKB result

$$
\psi(x)=O\left(x^{-3 / 2-\alpha / 2} \mathrm{e}^{-x^{4} / 4}\right) \quad x \rightarrow \infty .
$$

The ansatz (3.1) agrees with (3.2) for $\alpha=\alpha_{J}=-(2 l+1+4 J)$, a result which precisely matches the set (2.3). Let us now assume the existence of a solution to (2.8) of the form

$$
\phi(x)=x^{g_{1}} \mathcal{P}_{2 J-2}(x, E) \mathrm{e}^{-x^{2} / 2} .
$$

The relevant WKB asymptotic for the third-order ODE (2.8) is

$$
\phi(x)=O\left(x^{-1} \mathrm{e}^{-x^{2} / 2}\right) \quad x \rightarrow \infty .
$$

Comparing with (3.3) we find $g_{1}=1-2 J$, a very restricted set of values compared to (2.3). Starting from equation (2.17) leads to a similar conclusion. It is easy to check that when $g_{1}=1-2 J$ the ansatz (3.3) produces a single eigenfunction with eigenvalue $E=0$ for all odd integers $g_{1}$ satisfying $g_{0}<g_{1}<g_{2}$. However, away from these points we conclude that the eigenfunctions do not take the simple factorized form (3.3). Although other types of wavefunction factorization cannot be ruled out by this simple argument, we have checked that $\mathcal{H}_{3}$ is not one of the higher-order operators that appear in Turbiner's classification [3]. The surprise is that despite this, a subset of the eigenvalues of $\mathcal{H}_{3}$ with parameters (2.16) can be found algebraically as zeros of certain polynomials. 
Since we may not have identified the QES sector of $\mathcal{H}_{3}$ without the link to the isospectral problem $\mathcal{H}_{2}$, it is natural to ask whether there are any alternative ways to detect the appearance of this hidden quasi-exact solvability in our third-order equations or in other models.

To answer this question it is convenient to step back to [12] where, amongst other results, a series of full and partial isospectralities for $\mathcal{H}_{2}(\alpha, l) \equiv \mathcal{H}_{2}$ with boundary conditions (2.2) were observed. Four of them are summarized by the following diagram

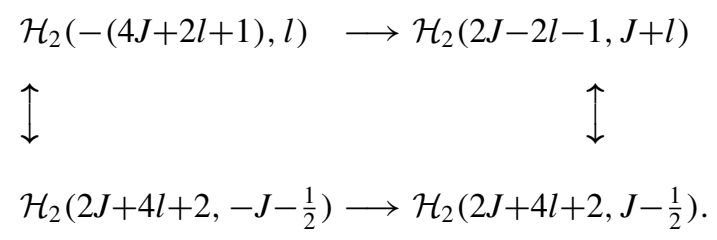

Vertical arrows correspond to eigenproblems that have exactly the same eigenvalues, while a horizontal arrow connects two problems that have the same eigenvalues up to the elimination of all the QES levels present in the left hand models. The two problems on the bottom row correspond to the same Schrödinger equation, and differ only in the boundary condition imposed at the origin. It follows from the diagram that the 'regular' eigenvalue problem for this equation, that with the behaviour $x^{J+1 / 2}$ at the origin, has exactly the same spectrum as the irregular problem with the $x^{-J+1 / 2}$ behaviour at the origin, with the exception of the first $J$ eigenvalues.

For general $l$, the eigenproblem $\mathcal{H}_{2}\left(2 J+4 l+2,-J-\frac{1}{2}\right)$ also does not appear in Turbiner's list of QES models [1]. Nonetheless, it is isospectral to the QES sextic Schrödinger problem and $J$ of its eigenvalues can be found exactly. In [12], after noticing an interesting symmetry in the recursion relation for the Bender-Dunne polynomials, it was (erroneously) stated that the appearance of QES eigenvalues in $\mathcal{H}_{2}\left(2 J+4 l+2,-J-\frac{1}{2}\right)$ corresponds also to a factorization of the eigenfunctions in the form (2.4). The latter statement can be checked using the simple consistency criteria introduced above. Setting

$$
\psi(x)=x^{-J+1 / 2} \mathcal{P}_{2 K-2}(x, E) \mathrm{e}^{-x^{4} / 4},
$$

at large $x$ the wavefunction behaves as $x^{-J-3 / 2+2 K} \mathrm{e}^{-x^{4} / 4}$ while the WKB prediction is $x^{-5 / 2-J-2 l} \mathrm{e}^{-x^{4} / 4}$. Hence (3.5) is a suitable ansatz only for

$$
l+1 / 2=-K, \quad K=1,2,3 \ldots
$$

Again, the constraint (3.6) is much stronger than (2.3) and we should conclude that for general values of $l$ the QES wavefunctions of $\mathcal{H}_{2}\left(2 J+4 l+2,-J-\frac{1}{2}\right)$ do not take a factorized form such as (3.5). We have not ruled out that the eigenfunctions can be written in terms of other elementary functions, a point to which we return at the end of this section. However, the key point is that with the standard techniques the quasi-exact solvability of $\mathcal{H}_{2}\left(2 J+4 l+2,-J-\frac{1}{2}\right)$ would not be evident and we would not know a priori how to determine the QES eigenvalues.

Returning to the question of how to detect quasi-exact solvability in such problems, we note that the eigenfunction $\psi(x, E, l)$ selected by the boundary conditions (2.2) is one of two solutions to (2.1), characterized by their small- $x$ behaviour

$$
\psi(x, E, l)=O\left(x^{l+1}\right), \quad \psi(x, E,-1-l)=O\left(x^{-l}\right) \quad x \rightarrow 0 .
$$

Provided the roots $l$ and $-1-l$ of the indicial equation do not differ by an integer, then the $\psi$ functions (3.7) are linearly independent. Moreover the solutions are automatically projectively trivial around the origin, by which we mean that for arbitrary $E$ the monodromy of $\psi(x, E, l)$ around $x=0$ is such that

$$
\psi\left(\mathrm{e}^{\mathrm{i} 2 \pi} x, E, l\right)=\mathrm{e}^{\mathrm{i} 2 \pi(l+1)} \psi(x, E, l) .
$$

The monodromy of $\psi(x, E,-1-l)$ follows via analytical continuation $l \rightarrow-1-l$. 
When $l=-J-\frac{1}{2}$ the roots of the indicial equation differ by $2 J$ and so for $J$ integer there is a 'resonance' between the two solutions $\psi(x, J) \equiv \psi\left(x, E,-J-\frac{1}{2}\right)$ and $\psi(x,-J) \equiv \psi\left(x, E, J-\frac{1}{2}\right)$. This pair is no longer a basis of solutions to $\mathcal{H}_{2}$ and a linearly independent solution $\widetilde{\psi}(x, E)$ to $\psi(x, E, J)$ must be constructed. In contrast to the regular solution $\psi(x,-J)$, the final solution will in general posses an additional logarithmic branch point at $x=0$ and thus the projective triviality property will therefore be lost:

$$
\widetilde{\psi}\left(\mathrm{e}^{\mathrm{i} 2 \pi} x, E\right) \neq \mathrm{e}^{\mathrm{i} 2 \pi(-J+1 / 2)} \widetilde{\psi}(x, E) .
$$

We now show that the eigenfunctions corresponding to the QES eigenvalues of $\mathcal{H}_{2}\left(2 J+4 l+2,-J-\frac{1}{2}\right)$ do not acquire logarithmic terms and so remain projectively-trivial. We suggest that this may be a means of detecting the hidden quasi-exact solvability of such models. To illustrate this we apply the Bender-Dunne method to $\mathcal{H}_{2}\left(2 J+4 l+2,-J-\frac{1}{2}\right)$ by setting

$$
\psi(x)=\mathrm{e}^{-x^{4} / 4} x^{-J+1 / 2} \sum_{n=0}^{\infty}\left(-\frac{1}{4}\right)^{n} \frac{Q_{n}(E, J, l)}{n !} x^{2 n} .
$$

In comparison with (2.4), the gamma function has been dropped from the denominator of (3.10) to ensure the coefficients in the series remain finite for all $n$ and (3.10) is a-by construction—projectively-trivial solution $\psi(x)$. Consequently the polynomials $Q_{n}(E)$ now satisfy

$$
(n-J) Q_{n}(E)=E Q_{n-1}(E)+16(n-1)(n+l-1 / 2) Q_{n-2}(E) .
$$

Setting $Q_{0}(E)=1$, the recursion relation defines $Q_{n}(E)$ in terms of $E$ and $J$ for $n<J$ just as before. The first difference occurs when $n=J$ since the lhs of (3.11) vanishes. The rhs is a $J$ thorder polynomial in $E$ which must be zero. Its roots therefore determine the QES eigenvalues $E_{n}, n=1, \ldots, J$. However, the recursion relation (3.11) has left $Q_{J}(E)$ unspecified and provided $l$ is such that $n+l-1 / 2 \neq 0$ the remaining coefficients do not factorize. In general, all $Q_{n>J}(E)$ will be a function of the unknown coefficient $Q_{J}(E)$. For example

$$
Q_{J+1}(E)=E Q_{J}(E)+16 J(J+l+1 / 2) Q_{J-1}(E) .
$$

Since $Q_{J-1}\left(E_{n}\right) \neq 0$, from (3.12) we see that the choice $Q_{J}\left(E_{n}\right)=0$ does not lead to a truncation of the series. More precisely, the resulting solution (3.10) will always have the desired monodromy properties but only for a precise value of the constant $Q_{J}\left(E_{n}\right)$ will it be asymptotically subdominant and satisfy the boundary condition at infinity.

Returning to the question of determining the exact eigenfunctions of the family $\mathcal{H}_{2}(2 J+4 l+2,-J-1 / 2)$, we note that for all odd integers $J$ one of the QES eigenvalues is $E=0$. In this case the eigenproblem can be solved in terms of a Whittaker function

$$
\psi(x)=\frac{2^{J / 4} \Gamma\left(\frac{3}{4}+\frac{J}{2}+\frac{l}{2}\right)}{\sqrt{\pi} x^{3 / 2}} W_{-\frac{J}{4}-\frac{1}{4}-\frac{l}{2}, \frac{J}{4}}\left(\frac{x^{4}}{2}\right),
$$

which exactly matches (3.10) when $E=0$ for the choice

$$
Q_{J}(0)=\frac{(-1)^{J / 2-1 / 2} 2^{5 J / 2} \sqrt{\pi} \Gamma\left(\frac{J}{2}+\frac{1}{2}\right) \Gamma\left(\frac{J}{2}+\frac{3}{4}+\frac{l}{2}\right)}{\Gamma\left(\frac{J}{2}\right) \Gamma\left(\frac{3}{4}+\frac{l}{2}\right)} .
$$

Noticing that for small $J$ the solution (3.13) when $l$ is an integer can be written in terms of Bessel functions multiplied by polynomials in $x$, we were motivated to try an ansatz of the form

$$
\psi(x)=x^{3 / 2-J} \sum_{n=0}^{\infty}\left(a_{n}(E) x^{2 n} K_{\frac{1}{4}}\left(\frac{x^{4}}{4}\right)+b_{n}(E) x^{2 n} K_{\frac{3}{4}}\left(\frac{x^{4}}{4}\right)\right)
$$


where $K_{n}(x)$ are modified Bessel functions of the second kind and $a_{n}(E), b_{n}(E)$ depend on $E, l$. Acting with $\mathcal{H}_{2}(2 J+4 l+2,-J-1 / 2)$ for integer $l$ on (3.15) and comparing powers of $x$, we found for $J=1$ that $E$ must be zero, the two series on the rhs of (3.15) truncated at $n=1+l$ and (3.15) reproduces (3.13) as anticipated. Setting $J=3$, we solved for the coefficients $\left\{a_{0}, a_{1}, \ldots, a_{3+l}, b_{0}, b_{1}, \ldots, b_{3+l}\right\}$ and found one solution with $E=0$ and two further solutions with eigenvalues $E= \pm 8 \sqrt{2+l}$, exactly reproducing the three solutions of $P_{3}(E)=0$. Repeating this process for odd $J$, we found that $(3.15)$ generates $J$ wavefunctions and constrains the QES eigenvalues to be solutions of $P_{J}(E)=0$. The series on the rhs of (3.15) truncated at $n=2(J+l)$.

Given the ansatz (3.15) unexpectedly generated not just the zero eigenvalues but all of the QES eigenvalues for $l$ integer when $J$ is an odd integer, we then checked if it also works for even integers $J$. It turns out the ansatz (3.15) indeed gave two solutions satisfying the boundary conditions (2.2) when $J=2$ provided $E= \pm 2 \sqrt{2} \sqrt{3+2 l}$, exactly matching (2.7). We find that the ansatz (3.15) worked for all integers $J, l \in \mathbb{N}^{+}$with the series on the rhs of (3.15) truncating at $n=2(J+l)$. However, at present we are not able to generalize this ansatz to non-integer values of $l$.

Differential operators that act invariantly on a subspace spanned by polynomials multiplied by special functions of either hypergeometric, Airy or Bessel type have been constructed in [17]. With the variable change $x=\sqrt{2} w^{1 / 4}$ and the gauge transformation $w^{-9 / 8} \mathcal{H}_{2} w^{5 / 8}$, we find $\mathcal{H}_{2}(2 J+l+2,-J-1 / 2)$ becomes proportional up to an additive constant to the differential operator $J_{5}^{+}$presented in [17] for the cases when $E=0$ and $l$ is an integer. To reproduce the solutions (3.13), the invariant subspace given in [17] for $J_{5}^{+}$must be extended to include Bessel functions multiplied by certain rational powers of $x$. We leave further details of these exact wavefunctions and the investigation of the cases when $l$ is not an integer to future work.

\section{Projective triviality and third-order QES models}

Continuing the discussion of the last section, we now show that the hidden QES sectors of $\mathcal{H}_{3}$ and $\mathcal{H}_{3}^{\dagger}$ can be detected by using the projective-triviality test discussed above on $\chi^{\dagger} \equiv \chi_{(0)}^{\dagger}$, one of the three linearly independent solutions $\left\{\chi_{(i)}^{\dagger}\right\}$ to (2.17). These solutions are characterized by their small- $x$ behaviour

$$
\chi_{(i)}^{\dagger}(x, \bar{E})=O\left(x^{g_{i}^{\dagger}}\right), \quad x \rightarrow 0
$$

where $g_{i}^{\dagger}=2-g_{i}(i=0,1,2)$ are the roots of the indicial equation. These solutions are projectively trivial around the origin

$$
\chi_{(i)}^{\dagger}\left(\mathrm{e}^{\mathrm{i} 2 \pi} x, \bar{E}\right)=\mathrm{e}^{\mathrm{i} 2 \pi g_{i}} \chi_{(i)}^{\dagger}(x, \bar{E})
$$

for $\left(g_{i}-g_{j}\right) \notin \mathbb{Z}$ with $i \neq j$. The QES eigenvalues of $\mathcal{H}_{3}, \mathcal{H}_{3}^{\dagger}$ appear when

$$
g_{0}=1 / 2+J-l, \quad g_{1}=1 / 2-2 J-l, \quad g_{2}=2+J+2 l,
$$

and

$$
g_{0}^{\dagger}=3 / 2-J+l, \quad g_{1}^{\dagger}=3 / 2+2 J+l, \quad g_{2}^{\dagger}=-J-2 l .
$$

Hence we have

$$
g_{0}^{\dagger}=g_{1}^{\dagger}-3 J, \quad J=1,2,3 \ldots
$$

Furthermore, for $l>-1 / 2$ and $J>0$ the ordering is $g_{2}^{\dagger}<g_{0}^{\dagger}<g_{1}^{\dagger}$, and we are in the presence of a resonance phenomena for the solutions $\left\{\chi_{i}^{\dagger}\right\}$. Again, this circumstance usually leads to the loss of the projective triviality property (4.2) due to the appearance of logarithmic 
contributions to the wavefunction. The analysis of section 3 suggests that imposing projective triviality on the eigenfunctions may be a way to identify the set of exactly known eigenvalues.

Instead of using a Bender-Dunne like ansatz for the wavefunction we will construct $\chi^{\dagger}$ perturbatively using Cheng's method [18]. The solution $\chi^{\dagger}$ to (2.17) is also a solution to the equation

$$
\chi^{\dagger}(x)=x^{2-g_{0}}+L\left[\left(x^{3}-\bar{E}\right) \chi^{\dagger}(x)\right]
$$

where

$$
L\left(x^{p}\right)=\frac{x^{p+3}}{\prod_{k}\left(p+1+g_{k}\right)}(k=0,1,2) .
$$

The function $\chi^{\dagger}$ can be considered as the $n \rightarrow \infty$ limit of a function $\chi^{\dagger(n)}$ constructed from $\chi^{\dagger(0)}=x^{2-g_{0}}$ using the following recursion relation

$$
\chi^{\dagger(n)}(x)=\chi^{\dagger(0)}(x)+L\left[\left(x^{3}-\bar{E}\right) \chi^{\dagger(n-1)}(x)\right] .
$$

After a single iteration, we have

$$
\chi^{\dagger(1)}=x^{2-g_{0}}\left(1-\frac{\bar{E} x^{3}}{\prod_{k}\left(3-g_{0}+g_{k}\right)}+\frac{x^{6}}{\prod_{j}\left(6-g_{0}+g_{k}\right)}\right) .
$$

Using (4.9) we can study the QES problem at $g_{0}-g_{1}=3 J$ when $J=1$. As $\left(g_{0}-g_{1}\right) \rightarrow 3$ the second term in the parenthesis on the rhs of (4.9) is in general divergent. However if the limit $\left(g_{0}-g_{1}\right) \rightarrow 3$ is taken simultaneously with $\bar{E} \rightarrow 0$ so that $\bar{E} /\left(3-g_{0}+g_{1}\right) \rightarrow C$ with $C$ finite, the final result is again finite and, up to this order in the perturbative expansion, $\chi^{\dagger}$ remains projectively trivial. The result $\bar{E}=0 \leftrightarrow P_{1}(E)=0$ corresponds precisely to the only exactly-solvable energy level at $J=1$. Further, the result of a second iteration is

$$
\begin{aligned}
\chi^{\dagger(2)}=x^{2-g_{0}} & \left(1-\frac{\bar{E} x^{3}}{\prod_{k}\left(3-g_{0}+g_{k}\right)}+\frac{x^{6}}{\prod_{k}\left(6-g_{0}+g_{k}\right)}\right. \\
& -\frac{\bar{E} x^{6}}{\prod_{k}\left(3-g_{0}+g_{k}\right)\left(9-g_{0}+g_{k}\right)}+\frac{\bar{E}^{2} x^{6}}{\prod_{k}\left(3-g_{0}+g_{k}\right)\left(6-g_{0}+g_{k}\right)} \\
& \left.-\frac{\bar{E} x^{9}}{\prod_{k}\left(6-g_{0}+g_{k}\right)\left(9-g_{0}+g_{k}\right)}+\frac{x^{12}}{\prod_{k}\left(6-g_{0}+g_{k}\right)\left(12-g_{0}+g_{k}\right)}\right) .
\end{aligned}
$$

At $J=1$, the potential divergences again disappear in the limit $\bar{E} /\left(3-g_{0}+g_{1}\right) \rightarrow C$, and it is easy to check that simultaneously all the subsequent $\chi^{\dagger(n)}$ remain finite. It is also possible to check that the solution corresponding to $C=0$ does not lead to the desired subdominant solution (4.6). The exact solution for $C=0$ is

$$
\left.\chi^{\dagger}(x)\right|_{C=0}=x^{2-g_{0}} F_{2}\left[\frac{1}{2}, 2-\frac{g_{0}}{2}, \frac{x^{6}}{216}\right] \quad(J=1, \bar{E}=0)
$$

which indeed grows exponentially as $x^{-1} \mathrm{e}^{x^{2} / 2}$. In order to find the proper wavefunction we use the asymptotics

$$
z^{\left(2-g_{0}\right) / 6}{ }_{0} F_{2}\left[\frac{1}{2}, 2-\frac{g_{0}}{2}, z\right] \sim \frac{\Gamma\left(2-\frac{g_{0}}{2}\right)}{2 \sqrt{3 \pi}} z^{-1 / 6} \mathrm{e}^{3 z^{1 / 3}} \quad z \rightarrow \infty,
$$

and introduce the linearly independent solution

$$
\chi_{1}^{\dagger}=z_{0}^{\left(5-g_{0}\right) / 6} F_{2}\left[\frac{3}{2}, \frac{5}{2}-\frac{g_{0}}{2}, z\right]
$$


which behaves asymptotically as

$$
\chi_{1}^{\dagger} \sim \frac{\Gamma\left(\frac{5}{2}-\frac{g_{0}}{2}\right)}{4 \sqrt{3 \pi}} z^{-1 / 6} \mathrm{e}^{3 z^{1 / 3}} \quad z \rightarrow \infty
$$

Thus, the asymptotically vanishing solution is

$\chi^{\dagger}(x)=x^{2-g_{0}}\left({ }_{0} F_{2}\left[\frac{1}{2}, 2-\frac{g_{0}}{2}, \frac{x^{6}}{216}\right]-\frac{\Gamma\left(2-\frac{g_{0}}{2}\right)}{3 \sqrt{6} \Gamma\left(\frac{5}{2}-\frac{g_{0}}{2}\right)} x_{0}^{3} F_{2}\left[\frac{3}{2}, \frac{5}{2}-\frac{g_{0}}{2}, \frac{x^{6}}{216}\right]\right)$.

The solution (4.15) corresponds to the choice $C=3 \sqrt{6} \Gamma\left(2-\frac{g_{0}}{2}\right) / \Gamma\left(\frac{3}{2}-\frac{g_{0}}{2}\right)$.

The case $J=2$ can be treated in a similar fashion: as $\left(g_{0}-g_{1}\right) \rightarrow 6$ the $x^{6}$ coefficient of (4.10) diverges. This singular behaviour can be avoided in the double limit

$$
\lim _{\bar{E} \rightarrow \bar{E}_{ \pm},\left(g_{0}-g_{1}\right) \rightarrow 6}\left(1+\frac{\bar{E}^{2}}{3\left(3-g_{0}+g_{1}\right)\left(3-g_{0}+g_{2}\right)}\right) /\left(6-g_{0}+g_{1}\right) \rightarrow C
$$

provided

$$
\bar{E}_{ \pm}= \pm 3 \sqrt{3} \sqrt{-2-g_{1}}= \pm 3 \sqrt{\frac{3}{2}} \sqrt{3+2 l} .
$$

The result (4.17) matches the exact energy levels (2.7) provided $E / \bar{E}=\kappa=4 /(3 \sqrt{3}$ ) (cf (2.11)). Further, the numerator of (4.16) is simply related to the Bender-Dunne polynomial $P_{2}(E)$ (see below) and it is possible to argue that there always exists a value of $C$ such that the wavefunction decays exponentially at large $x$. This proves, therefore, that the roots of the Bender-Dunne polynomials $P_{2}(E)$ are indeed part of the spectrum of the dual pair (2.8), (2.17).

More generally, for any $\left\{g_{i}\right\}$ the full Cheng solution can be written in the form

$$
\chi^{\dagger}(x)=x^{2-g_{0}}\left(\sum_{n=0}^{\infty} \frac{(-1)^{n} \bar{P}_{n}(\bar{E}) x^{3 n}}{\prod_{k=0}^{2}\left(3 n-g_{0}+g_{k}\right)}\right)
$$

where $\bar{P}_{n}(\bar{E})$ are degree $n$ polynomials in $\bar{E}$ that satisfy

$$
\bar{P}_{n}(\bar{E})=\bar{E} \bar{P}_{n-1}(\bar{E})+\prod_{k=0}^{2}\left(3(n-1)-g_{0}+g_{k}\right) \bar{P}_{n-2}(\bar{E})
$$

with $\bar{P}_{0}=1, \bar{P}_{1}=\bar{E}$. Restricting $\left\{g_{i}\right\}$ to the QES points (4.3), the recursion relation (4.19) matches the Bender-Dunne recursion relation (2.5) with $\kappa^{n} \bar{P}_{n}(\bar{E})=P_{n}(E)$.

\section{Other models and conclusions}

In this paper we have reported some progress toward a more complete understanding of the spectral equivalence between the sextic anharmonic oscillator (2.1) and the dual pair of third-order ODEs (2.8) and (2.17), and the nature of the quasi-exact solvability of the thirdorder problems that is thereby induced. Although more work will be needed to complete the picture, and more generally to understand the emergence of standard and hidden quasiexact solvability in dual pairs $\left\{\mathcal{H}, \mathcal{H}^{\dagger}\right\}$ of higher-order differential operators, we think that the concept of projective triviality should be a useful tool in detecting QES sectors. In addition to the examples discussed in section 3 and section 4, we have discovered that hidden quasi-exact solvability is a property shared by many other models. For example, we have applied the same analysis to the $n$th order differential equations introduced in [19]

$\left[(-1)^{n+1} D\left(g_{n-1}-(n-1)\right) D\left(g_{n-2}-(n-2)\right) \cdots D\left(g_{0}\right)+x^{n M}\right] \psi(x)=E \psi(x)$ 
where

$$
D(g)=\left(\frac{\mathrm{d}}{\mathrm{d} x}-\frac{g}{x}\right), \quad \sum_{i=0}^{n-1} g_{i}=\frac{n(n-1)}{2}
$$

and the boundary conditions are

$$
\left.\psi\right|_{x \rightarrow 0}=O\left(x^{g_{1}}\right), \quad \psi=O\left(x^{(1-n) M / 2} \mathrm{e}^{-x^{M+1} /(M+1)}\right) \text { as } x \rightarrow \infty
$$

with $g_{0}<g_{1}<\ldots<g_{n-1}$. These directly generalize the problems $\mathcal{H}_{2}$ with $\alpha=0$ and $\mathcal{H}_{3}$. By imposing projective triviality on the wavefunctions of the adjoint problems at the resonant points $g_{0}-g_{i}=n J$ for $M, J \in \mathbb{N}$, we found the Cheng solutions for the adjoint problems are

$$
\chi^{\dagger}(x)=x^{n-1-g_{0}}\left(\sum_{m=0}^{\infty} \frac{(-1)^{m} P_{m}(E) x^{m n}}{\prod_{j=1}^{m} \prod_{k=0}^{n-1}\left(n j-g_{0}+g_{k}\right)}\right)
$$

where the corresponding Bender-Dunne polynomials satisfy

$P_{m}(E)=E P_{m-1}(E)-(-1)^{M} \prod_{j=1}^{M} \prod_{k=0}^{n-1}\left(n(j+m-M-1)-g_{0}+g_{k}\right) P_{m-1-M}(E)$.

The QES eigenvalues are the $J$ roots of $P_{J}(E)=0$ and, in general, the associated wavefunctions do not have an elementary form. As noted in section 3 for the second-order models, the nonQES part of the spectrum is precisely the spectrum of the same differential equation subject to a boundary condition that imposes regular behaviour of the wavefunction at the origin.

Finally, we should reiterate that for all the QES models encountered in this paper when $g_{0}-g_{i}=n J$, the ordering $g_{0}<g_{1}<g_{2}<\cdots$ of the solutions of the indicial equations is not fulfilled. Hence, the norm (2.20) of the exactly-solvable states $\sqrt{\langle n \mid n\rangle}$ is divergent and the corresponding radial eigenvalue problem is always 'irregular'. Higher-order differential equations of the form (5.1) have recently been studied in the context of $\mathcal{P} \mathcal{T}$ symmetric quantum mechanics and its generalizations for even $n$ in [20,21]. Additional motivation for the further study of higher-order eigenproblems of the type considered in this paper comes from their relevance to particular integrable quantum field theories, via the ODE/IM correspondence $[19,13,22-24]$.

\section{Acknowledgments}

RT thanks Miloslav Znojil for useful discussions and kind encouragement to finish this paper. This project was partially supported by an INFN grant PI11, EPSRC grant EP/G039526/1, the Leverhulme Trust and the Italian MIUR-PRIN contract 2009KHZKRX-007 'Symmetries of the Universe and of the Fundamental Interactions'.

\section{References}

[1] Turbiner A V 1988 Quasi-exactly-solvable problems and sl(2) algebra Commun. Math. Phys. 118467

[2] Turbiner A V and Ushveridze A G 1987 Spectral singularities and the quasi-exactly-solvable problem Phys. Lett. A 126181

[3] Turbiner A V 1992 Lie algebras and polynomials in one variable J. Phys. A: Math. Gen. 25 L1087

[4] Dolya S N and Zaslavskii O B 2000 Quantum anharmonic oscillator and quasi exactly solvable Bose systems J. Phys. A: Math. Gen. 33 L369 (arXiv:quant-ph/0009071)

[5] Alvarez G, Finkel F, Gonzalez-Lopez A and Rodrguez M A 2002 Quasi-exactly solvable models in nonlinear optics J. Phys. A: Math. Gen. 358705

[6] Alvarez G and Alvarez-Estrada R F 2004 Third-order equation for harmonic generation: complex canonical transformation and JWKB solution J. Phys. A: Math. Gen. 372611 
[7] Lee Y-H, Yang W-L and Zhang Y-Z 2010 Polynomial algebras and exact solutions of general quantum nonlinear optical models: I. Two-mode boson systems J. Phys. A: Math. Theor. 43185204 (arXiv:1002.0677 [math-ph])

[8] Lee Y-H, Yang W-L and Zhang Y-Z 2010 Polynomial algebras and exact solutions of general quantum nonlinear optical models: II. Multi-mode boson systems J. Phys. A: Math. Theor. 43375211 (arXiv:1004.1235 [math-ph])

[9] Dorey P and Tateo R 2000 Differential equations and integrable models: the $S U$ (3) case Nucl. Phys. B 571583 Dorey P and Tateo R 2001 Erratum to: "Differential equations and integrable models: the $S U$ (3) case" [Nucl. Phys. B 571 (2000) 583] Nucl. Phys. B 603582 (arXiv:hep-th/9910102)

[10] Bender C M and Dunne G V 1996 Quasi-exactly solvable systems and orthogonal polynomials J. Math. Phys. 376 (arXiv:hep-th/9511138)

[11] Suzuki J 2001 Functional relations in Stokes multipliers-fun with $x^{6}+\alpha x^{2}$ potential J. Stat. Phys. 1021029 (arXiv:quant-ph/0003066)

[12] Dorey P, Dunning C and Tateo R 2001 Spectral equivalences, Bethe ansatz equations, and reality properties in $\mathcal{P} \mathcal{T}$-symmetric quantum mechanics J. Phys. A: Math. Gen. 345679 (arXiv:hep-th/0103051)

[13] Dorey P, Dunning C, Masoero D, Suzuki J and Tateo R 2007 Pseudo-differential equations, and the Bethe Ansatz for the classical Lie algebras Nucl. Phys. B 772249 (arXiv:hep-th/0612298)

[14] Dorey P, Dunning C, Masoero D, Suzuki J and Tateo R 2009 ABCD and ODEs New Trends in Mathematical Physics ed V Sidoravičins (Berlin: Springer) pp 685-95 (arXiv:0704.2109 [hep-th])

[15] Bender C M, Boettcher S and Meisinger P $1999 \mathcal{P} \mathcal{T}$-symmetric quantum mechanics J. Math. Phys. 402201 (arXiv:quant-ph/9809072)

[16] Curtright $\mathrm{T}$ and Mezincescu L 2007 Biorthogonal quantum systems J. Math. Phys. 48092106 (arXiv:quant-ph/0507015)

[17] Dolya S N 2008 Quasi-exactly solvable models based on special functions J. Math. Phys. 49053524

[18] Cheng H 1962 Meromorphic property of the $S$ matrix in the complex plane of angular momentum Phys. Rev. 127647

[19] Dorey P, Dunning C and Tateo R 2000 Differential equations for general SU(n) Bethe ansatz systems J. Phys. A: Math. Gen. 338427 (arXiv:hep-th/0008039)

[20] Bender C M and Hook D W 2008 Exact isospectral pairs of PT-symmetric Hamiltonians J. Phys. A: Math. Theor. 41244005 (arXiv:0802.2910 [hep-th])

[21] Bender C M and Hook D W 2012 Universal spectral behavior of $x^{2}(i x)^{\epsilon}$ potentials arXiv:1205.4425 [hep-th]

[22] Dorey P and Tateo R 2000 Differential equations and integrable models: the SU (3) case Nucl. Phys. B 571583 (arXiv:hep-th/9910102)

[23] Suzuki J 2000 Functional relations in Stokes multipliers and solvable models related to $U_{q}\left(A_{n}^{(1)}\right) J$. Phys. A: Math. Gen. 333507 (arXiv:hep-th/9910215)

[24] Bazhanov V V, Hibberd A N and Khoroshkin S M 2002 Integrable structure of $W_{3}$ conformal field theory, quantum Boussinesq theory and boundary affine Toda theory Nucl. Phys. B 622475 (arXiv:hep-th/0105177) 